\title{
Case Report \\ Surgical Treatment of a Chronic Brain Abscess and Growing Skull Fracture in a Dog
}

\author{
Amy W. Hodshon, ${ }^{1}$ Jill Narak, ${ }^{2}$ Linden E. Craig, ${ }^{3}$ and Andrea Matthews ${ }^{4}$ \\ ${ }^{1}$ Department of Small Animal Clinical Sciences, College of Veterinary Medicine, The University of Tennessee, \\ 2407 River Drive, Knoxville, TN 37996, USA \\ ${ }^{2}$ Department of Clinical Sciences, Auburn University, Auburn, AL 36849, USA \\ ${ }^{3}$ Department of Biomedical and Diagnostic Sciences, College of Veterinary Medicine, The University of Tennessee, \\ 2407 River Drive, Knoxville, TN 37996, USA \\ ${ }^{4}$ Antech Imaging Services, Charlottetown, PE, Canada C1A $1 B 6$
}

Correspondence should be addressed to Jill Narak; narakjc@auburn.edu

Received 6 April 2015; Accepted 24 May 2015

Academic Editor: Sheila C. Rahal

Copyright (C) 2015 Amy W. Hodshon et al. This is an open access article distributed under the Creative Commons Attribution License, which permits unrestricted use, distribution, and reproduction in any medium, provided the original work is properly cited.

A 2-year-old female spayed Miniature Dachshund was presented for seizures and right prosencephalic signs. A multiloculated, ring-enhancing mass in the right cerebrum associated with dilation of the right lateral ventricle and brain herniation was seen on magnetic resonance imaging. An irregular calvarial defect with smoothly scalloped edges was seen overlying the mass on computed tomography. The mass was removed via craniectomy and was diagnosed as a chronic brain abscess caused by Peptostreptococcus anaerobius. The patient was maintained on antibiotics for 12 weeks. Follow-up MRI performed 14 weeks after surgery confirmed complete removal of the abscess as well as a contrast-enhancing collection of extra-axial material consistent with a chronic subdural hematoma. The neurologic abnormalities, including seizures, have improved in the 44 months since surgery. Brain abscesses in dogs can have an insidious clinical course prior to causing serious neurologic deterioration. Ventricular entrapment by an intracranial mass can contribute to acute neurologic decline. If surgically accessible, outcome following removal of a brain abscess can be excellent; aerobic and anaerobic bacterial culture should be performed in these cases. Subdural hematoma can occur following removal of a large intracranial mass. Growing skull fractures can occur in dogs but may not require specific surgical considerations.

\section{Introduction}

Brain abscesses are a relatively uncommon cause of neurologic disease in dogs. Surgical treatment has been reported in one dog and eight cats, each with a brain abscess suspected to be caused by a bite wound received within 14 days of presentation [1-3]. In contrast, this report describes the surgical treatment of a dog with a chronic, mature brain abscess. Additional interesting features of this case that are presented and discussed include the presence of a growing skull fracture, compartmentalized hydrocephalus of the lateral ventricle, and a suspected chronic subdural hematoma on postoperative imaging.

\section{Case Presentation}

A 2-year-old female spayed Miniature Dachshund was presented for a 5-day history of circling to the right and blindness in the left eye. This had been preceded by 2 weeks of lethargy and reluctance to traverse stairs for which she was receiving carprofen (unknown dose). The dog had a history of generalized seizures that had begun 7 months earlier and occurred about twice monthly; she had been receiving phenobarbital ( $8 \mathrm{mg}$ by mouth twice daily) for the previous 6 months. The dog was obtained from a breeder at 7 months of age; she had a scar on her head at that time that according to the breeder had always been present. The owner reported 
that occasionally the area of the scar would swell for a period of time and then go back down.

On presentation, the patient was quiet but responsive with normal vital parameters. General physical exam was normal other than an irregular scar present over the right frontal bone; a bony defect with underlying soft, compressible tissue was palpable under the scar. On neurologic exam the dog's mentation was depressed, and she circled to the right with a normal gait. Cranial nerve exam revealed blindness in the left visual field with normal pupillary light reflexes. Postural reactions were delayed on the left side and normal on the right. Spinal reflexes were normal. Pain was elicited on palpation of the cranial cervical spine and head. These findings were consistent with a structural lesion in the right forebrain. Primary differential diagnoses included a brain abscess, other inflammatory diseases (infectious or sterile), and a neoplastic mass.

A complete blood count and serum chemistry were within normal limits. The patient was anesthetized, and magnetic resonance imaging (MRI) of the brain was performed (1.0 Tesla Magnetom Harmony, Siemens Medical Solutions, Malvern, PA). A mass lesion was seen in the right cerebrum located under a defect in the dorsal calvarium. Within the mass were several pockets of T2-hyperintense material incompletely suppressed on T2-weighted fluid attenuated inversion recovery (FLAIR) (Figures 1 (a) and $1(\mathrm{~b})$ ). These pockets were surrounded by a T2-hypointense, T1-isointense rim. There was significant $\mathrm{T} 2 \mathrm{~W}$ hyperintensity within the right cerebral white matter, consistent with vasogenic edema. The rostral horn of the right lateral ventricle was adjacent to the caudal extent of the mass, and caudal to this the ventricle was markedly dilated and surrounded by a rim of T2W and FLAIR hyperintensity (Figure 1(c)). The cumulative mass effect was causing significant shifts in brain parenchyma, including subfalcine herniation (Figures 1(d) and 2). The cervical spinal cord was T2-hyperintense, especially dorsally starting at C2-C3, suggestive of syringohydromyelia formation. On the $\mathrm{T} 2{ }^{*}$-weighted gradient-echo sequence, there were several irregular areas of signal void associated with the mass, likely indicative of hemorrhage or mineral fragment (Figure 1(e)). The periphery of the mass strongly enhanced immediately following administration of intravenous (IV) paramagnetic contrast agent (Magnevist, Bayer Healthcare Pharmaceuticals Inc., Wayne, NJ; $0.1 \mathrm{mmol} / \mathrm{kg}$ ). This enhancement clearly delineated at least three distinct pockets of T1-hypointense fluid (Figure 1(f)). No ependymal or diffuse meningeal contrast enhancement was seen.

A computed tomography (CT) scan (Philips Brilliance 40, Philips Healthcare, Andover, MA) was performed following the MRI. A large area of hypodense parenchyma in the right cerebral hemisphere causing a significant midline shift toward the left was seen (Figure 3(a)). There was a large, irregular, chevron-shaped defect in the right frontal bone with a smaller triangular-shaped bone flap overlying it; this bone flap extended cranially tangential to the convexity of the skull (Figure 3(b)). The margins of the bone flap and the underlying skull defect were smoothly scalloped. The defect did not extend into the frontal sinus. A small mineral fragment was also noted within the intracranial mass
(Figure 3(a)). Postcontrast CT was not performed. Based on examination and imaging findings, a cerebral abscess was suspected, and the patient was taken immediately to surgery.

The patient received $1 \mathrm{~g} / \mathrm{kg}$ mannitol IV over about 20 minutes following MRI; perioperative antibiotics were withheld. A modified right rostrotentorial craniectomy was performed centered over the bony defect. The triangular flap of bone was removed using rongeurs and was noted to be spongier than normal bone. The cerebrum under the bony defect was covered with tenacious fibrous tissue; distinct dura mater was difficult to identify. The edges of the calvarial defect were freshened, and the dura and fibrous tissue overlying the protruding brain tissue were incised and removed.

An ultrasonic aspirator (Sonastar, Mixonix, Inc., Farmingdale, NY) was inserted to a premeasured depth to reach the contrast-enhancing mass seen on MRI; however, the mass's firm consistency precluded use of the aspirator. Gentle probing with lint-free eye spears (Eagle Labs, Rancho Cucamonga, CA) allowed movement of the intra-axial mass, which was green/brown, firm, and distinct from the surrounding brain parenchyma. The mass was gently dissected free of surrounding tissue using an ophthalmic spatula and removed. A small bone chip was embedded in the mass, and the center was cavitated and had a foul odor on cut section. Half of the removed tissue was submitted for aerobic, anaerobic, and fungal tissue culture. The remaining tissue was fixed in $10 \%$ formalin for histopathologic analysis. During removal of the mass, the right lateral ventricle was inadvertently entered, presumably at the site where the two were adjacent; the ependymal lining and choroid plexus were visible, and cerebrospinal fluid flowed into the surgical field. The ventricular tissue was gently replaced into the parenchymal defect created by removal of the mass in as normal an anatomic orientation as possible.

Following removal of the mass, $20 \mathrm{mg} / \mathrm{kg}$ enrofloxacin was given IV over one hour. Once hemostasis was achieved, the surgical site was copiously lavaged with sterile saline, and the durectomy was covered with swine intestinal submucosa (SurgiVet, Dublin, OH). Polypropylene mesh (C.R. Bard, Inc., Warwick, RI) was cut slightly larger than the craniectomy and was sutured to the surrounding periosteum. The subcutaneous tissues and skin were closed in a routine fashion.

The patient recovered from anesthesia uneventfully. Postoperative care included a fentanyl and lidocaine infusion (titrated for analgesia), enrofloxacin $(20 \mathrm{mg} / \mathrm{kg}$ IV once daily), clindamycin (12 mg/kg IV twice daily), famotidine $(0.5 \mathrm{mg} / \mathrm{kg}$ IV twice daily), phenobarbital $(2.5 \mathrm{mg} / \mathrm{kg} \mathrm{IV}$ twice daily), maintenance IV fluids, and standard monitoring and nursing care. The morning after surgery, the patient was sedated but was responsive and nonpainful. She was ambulatory with mild left-sided hemiparesis 24 hours following surgery (likely due to peracute disruption of the right frontoparietal lobe at the time of surgery). Postoperative complications included a seroma at the surgery site and two instances of regurgitation on the first day of oral feeding (48 hours after surgery); these were treated with intermittent warm compresses and metoclopramide $(0.25 \mathrm{mg} / \mathrm{kg}$ orally every 6 hours), respectively. A five-day course of antiinflammatory prednisone $(0.25 \mathrm{mg} / \mathrm{kg}$ orally twice daily) was 


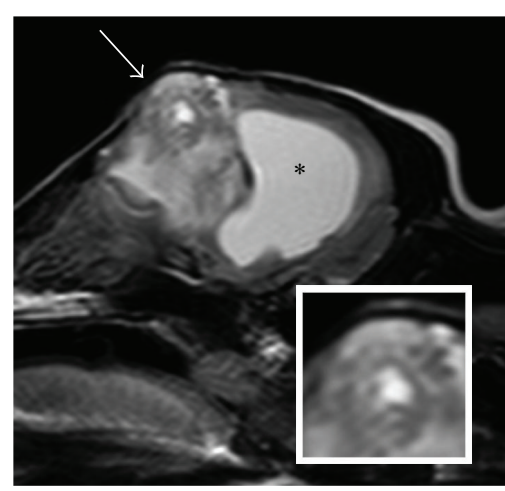

(a)

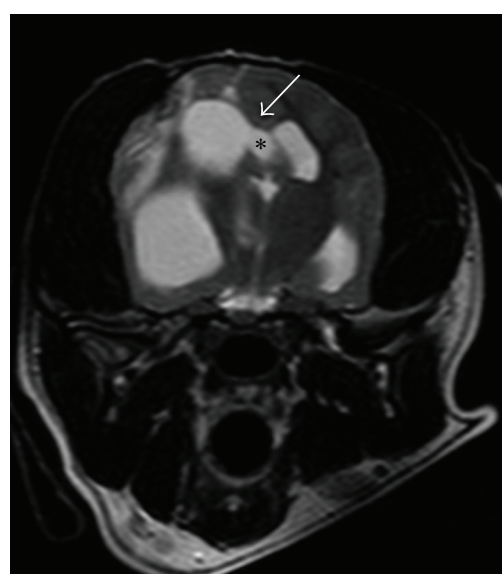

(d)

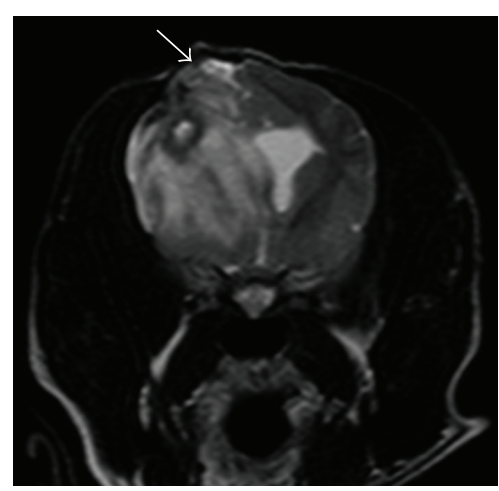

(b)

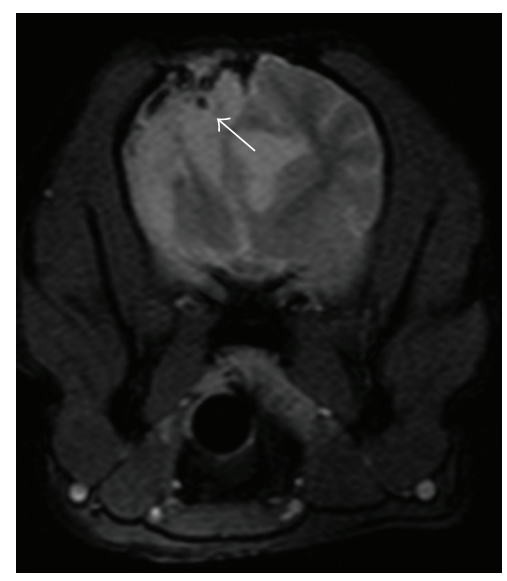

(e)

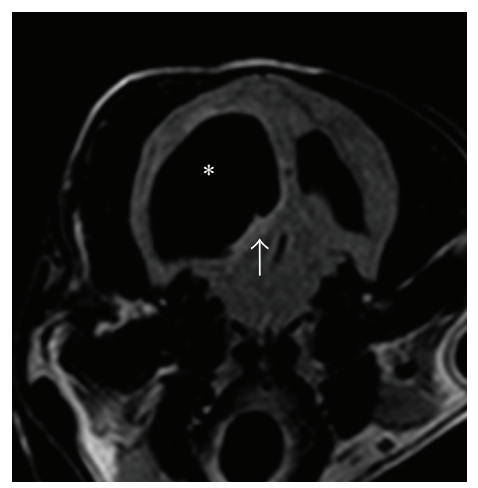

(c)

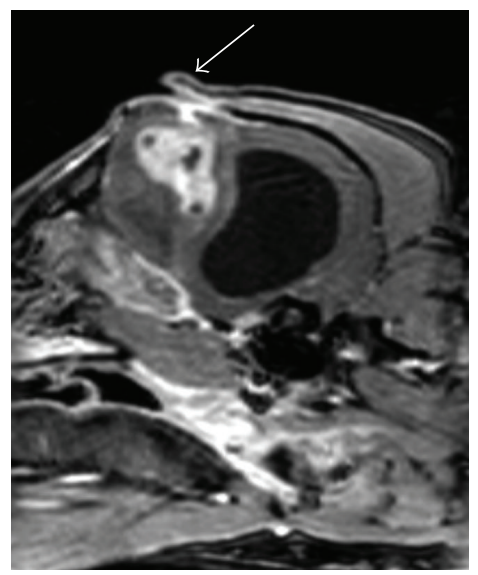

(f)

FIGURE 1: Preoperative magnetic resonance (MR) images of the head. (a) T2-weighted right parasagittal image. There is a predominantly T2-hyperintense mass in the right cerebrum underlying a calvarial defect (arrow) and significant dilation of the right lateral ventricle (asterisk). Inset: note the multiple rims of alternating intensity surrounding the T2-hyperintense center, not suppressed on a T2-weighted FLAIR sequence. (b) T2-weighted transverse image at the level of the optic chiasm. The mass is causing midline shift toward the left and brain herniation through the calvarial defect (arrow). (c) T2-weighted FLAIR transverse image at the level of the midbrain. There is hyperintensity immediately surrounding the markedly dilated right lateral ventricle (asterisk) and descending transtentorial herniation of the right parahippocampal gyrus (arrow). (d) T2-weighted transverse image at the level of the caudal thalamus. There is subfalcine herniation of the right cingulate gyrus (arrow) and part of the right lateral ventricle (asterisk). (e) T2 ${ }^{*}$-weighted transverse image at the level of the optic chiasm. There are several irregular areas of signal void, one (arrow) that corresponds with the bone fragment seen on CT imaging. (f) Postcontrast T1-weighted gradient-recalled echo right parasagittal image. There are three pockets of T1-hypointense material surrounded by a strongly contrast-enhancing rim. Note the elevated bone flap that incompletely spans the calvarial defect (arrow).

initiated four days after surgery. No seizures were observed during the 5 days the patient was hospitalized. At the time of discharge the neurologic exam was similar to that on presentation. Oral clindamycin $(12 \mathrm{mg} / \mathrm{kg}$ twice daily) and enrofloxacin $(20 \mathrm{mg} / \mathrm{kg}$ once daily) were continued at home as well as the maintenance dose of phenobarbital.

Anaerobic culture of tissue obtained at surgery yielded growth of Peptostreptococcus anaerobius. Aerobic bacterial and fungal cultures were negative. Microscopically, the central cavity of the mass contained a linear fragment of necrotic bone (Figure 4(a)) and innumerable Gram-positive bacterial cocci (Figure 4(b)). The tissue immediately surrounding the cavity contained abundant neutrophils and fewer macrophages; the inflammation became more lymphoplasmacytic with fibroblasts and large bands of fibrosis at the periphery (Figure 4(c)). The most peripheral neuropil in the submitted sample had diffuse gliosis and perivascular cuffs of lymphocytes and plasma cells (Figure 4(d)).

The patient returned two weeks after surgery for a recheck exam, and the owners reported marked improvement in her attitude at home. She continued to circle to the right and was blind in her left visual field. She had not had any seizures since surgery. Postural reactions on the left were improved though still not normal, and cervical pain was no longer present. At this point enrofloxacin was discontinued based on the identification of an anaerobic organism, and metronidazole therapy (10 $\mathrm{mg} / \mathrm{kg}$ by mouth twice daily) was instituted.

Peptostreptococcus isolates were submitted to the University of Illinois Veterinary Diagnostic Laboratory (Urbana, IL) for susceptibility testing and were found to be resistant to metronidazole but susceptible to clindamycin. Once these results were received, metronidazole therapy was 


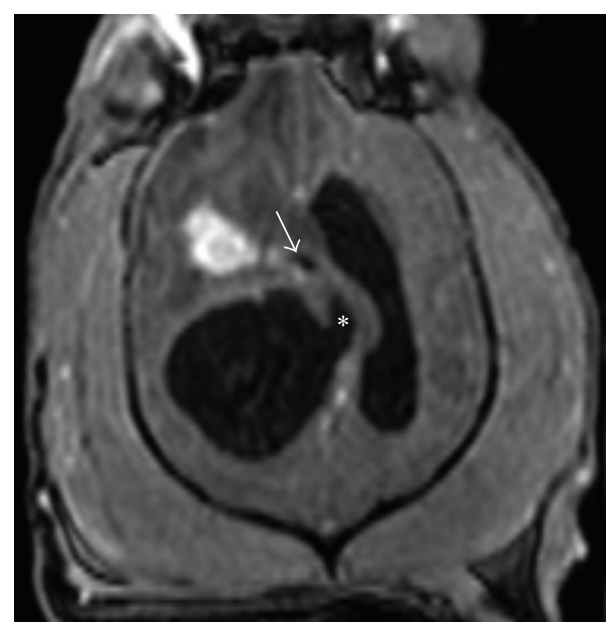

FIGURE 2: Preoperative postcontrast T1-weighted dorsal MR image of the head. There is subfalcine herniation of the right lateral ventricle (asterisk) with subsequent suspected isolation of the lateral ventricle from the third ventricle (arrow).

discontinued, and the patient was continued on clindamycin alone in addition to phenobarbital.

The patient returned approximately 14 weeks after surgery. She had had no known seizures since surgery, and mentation and activity level were normal. She was still receiving phenobarbital, but clindamycin had been discontinued without instruction about two weeks earlier (the intended course was at least 16 weeks long). She continued to circle occasionally to the right, though the owners felt this had improved. Left-sided menace and postural reaction deficits persisted.

The patient was anesthetized for a brain MRI, and there was marked T2W hyperintensity in the rostral right cerebral hemisphere with loss of normal tissue architecture. The right lateral ventricle was no longer dilated, but its shape was abnormal and came to a point dorsally in the area of the previous abscess (presumably where it was penetrated during surgery) (Figure 5(a)). A midline shift was present, but it was toward the right due to parenchymal loss on that side (Figure 5(b)). Within the right cerebral cortex and white matter, there were pockets of $\mathrm{T} 2 \mathrm{~W}$ hyperintensity suppressed on T2W FLAIR images but did not correspond with the lateral ventricle, suggestive of hydrocephalus ex vacuo (Figure 5(b)). The cervical spinal cord appeared normal.

There was a peripheral rim of heterogeneously T2hyperintense, T1-hypointense material conforming to the surface of the right cerebral hemisphere that extended ventrally over the piriform lobe and caudally over the surface of the occipital lobe (Figure 5(c)); in areas this rim had layers of mixed signal on T2-weighted images (Figure 5(d)). Areas of the rim showed susceptibility artifact on $\mathrm{T} 2{ }^{*}$-weighed sequences, consistent with hemorrhage (Figure 5(e)). The majority of this plaque-like rim, especially its outer border, was strongly and homogenously enhanced following contrast administration. The enhancement extended along the entire convexity of the hemisphere (Figures $5(\mathrm{f})$ and $5(\mathrm{~g})$ ) with sharp margins along the plane of the falx cerebri and tentorium cerebelli; it did not extend into the parenchyma.

A sample of cerebrospinal fluid obtained from the cerebellomedullary cistern contained two nucleated cells (mononuclear monocytoid cells and small lymphocytes) and 2 red blood cells per microliter; protein content was mildly elevated at $42.2 \mathrm{mg} / \mathrm{dL}$. Aerobic and anaerobic bacterial cultures were negative.

At the time of writing (44 months after surgery), the patient is reportedly doing well at home. Blindness in the left visual field and occasional circling to the right persist, but activity level is normal, and personality is normal to slightly more reserved than prior to presentation. She is maintained on $8.2 \mathrm{mg}$ phenobarbital twice daily and has a seizure approximately once every four months.

\section{Discussion}

CT and MRI characteristics of brain abscesses have been described in humans and domestic animals [1-8]. The MR appearance of the abscess in this case is typical of a mature brain abscess, including pockets of T2-hyperintense material surrounded by a T2-hypointense rim, large amounts of vasogenic edema, and strong peripheral contrast enhancement [7-9]. One apparently distinct and as yet unexplained MRI feature of brain abscesses that was present in this case is the appearance of multiple rims on T2-weighted sequences (Figure 1(a) and inset). When this feature is present, the central hyperintense cavity of the abscess is bordered by concentric rings that are hypo-, then hyper-, and then hypointense; hyperintense edema then surrounds the lesion, giving it a target appearance. There is no known histopathologic correlate to these rings, though they may represent different layers of necrotic debris and inflammation or may be caused by an unknown artifact $[7,10]$.

The imaging appearance of the abscess in this case was consistent with a multiloculated abscess. In human case series, up to $20 \%$ of brain abscesses are multiloculated [1115]. This is thought to result from poor abscess encapsulation. In general, abscesses tend to grow toward white matter and away from well-vascularized gray matter, and the medial wall of the capsule tends to be thinner, which allows "daughter abscesses" to bleb off. While not known to be prognostically significant in people, multiloculated abscesses more often require surgical excision rather than CT-guided aspiration to achieve resolution $[13,16]$.

The mortality rate in humans with brain abscesses has declined significantly since the advent of CT and is currently between 8 and 25\% [15, 17]. Abscesses are treated surgically (by excision or drainage) whenever possible; indications to delay or avoid surgical excision include multiple distantly located abscesses, abscesses in deep or vital brain structures, and concurrent meningitis or ependymitis $[16,18,19]$. An additional indication to promptly perform surgery in the case reported here was the presence of obstructive hydrocephalus of the right lateral ventricle, as evidenced by severe ventricular dilation with periventricular T2 hyperintensity (consistent with interstitial edema) on the MRI. This is an unusual manifestation of focal hydrocephalus known as 


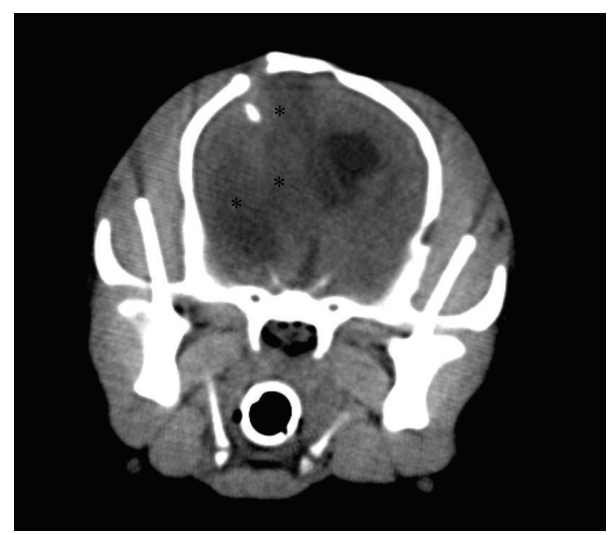

(a)

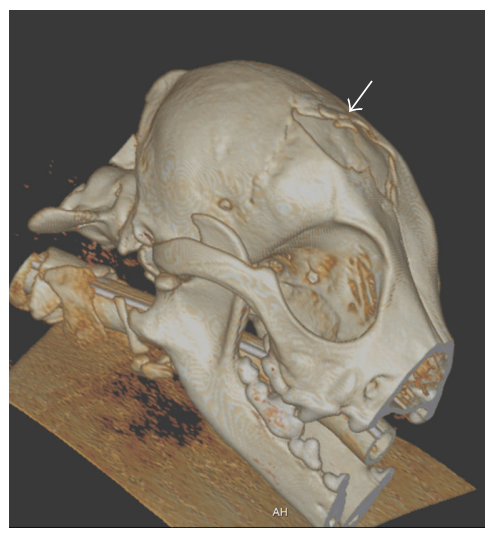

(b)

Figure 3: Preoperative, precontrast computed tomography (CT) images of the head. (a) Image in a soft tissue window at the level of mandibular rami. There is midline shift toward the left caused by a large hypodensity in the right cerebrum (asterisks), a calvarial defect, and a mineral fragment within the brain parenchyma. (b) Three-dimensional CT reconstruction of the skull in a bone window. Note the smoothly scalloped edges of the elevated bone flap (arrow) and calvarial defect as well as the size discrepancy between the two.

a "trapped ventricle" $[20,21]$. In this dog's case the temporal horn of the lateral ventricle was "trapped"; this can occur when the interventricular foramen on one side is occluded and to the authors' knowledge has not been previously reported in a dog. When the interventricular foramen is occluded, the cerebrospinal fluid that is continually produced by the choroid plexus of the lateral ventricle accumulates. Hypertensive hydrocephalus develops, and the ventricle itself can behave as a space-occupying mass. In people, trapped temporal horn has been reported secondary to multiple causes including extraventricular compression by a tumor, intraventricular masses, ventricular trauma, and intraventricular hemorrhage [22-24]. A trapped ventricle requires expedient surgical intervention to either remove the cause of obstruction or shunt CSF away from the ventricle [21, 25, 26]. The apparent cause of obstruction in the case presented here was the extraventricular mass effect created by the abscess and surrounding edema in the area of the right interventricular foramen (Figures 1(d) and 2). We did not measure intraventricular pressure to document hypertension, but eventual occlusion of the interventricular foramen by a slowly growing abscess may explain this patient's relatively rapid neurologic deterioration following the otherwise insidious clinical course for the first 18 to 24 months of her life.

Another interesting feature of this case that, to our knowledge, has not previously been reported as a natural finding in a dog is the presence of what is termed a "growing skull fracture." This is a well-described but incompletely understood injury seen in children who suffer head trauma while both the skull and brain are rapidly growing (usually at less than one year and rarely more than three years of age) [27]. Four requisite features of a growing skull fracture have been described: (1) skull fracture at a young age, (2) dural tear, (3) brain injury underlying the fracture, and (4) subsequent enlargement of the fracture to form a cranial defect [27]. Children with this condition often present several weeks to years following the initial injury; the most common complaint is a pulsatile scalp mass, although neurologic deficits can also be present. The bone edges are usually scalloped and thickened and are occasionally elevated away from the brain surface as they were in the case presented here [28].

The pathogenesis of growing skull fractures remains unclear, but current theories are based on abnormal lines of force in the area of the dural and bony defect caused by the physiologic pulsations of the brain and ventricular cerebrospinal fluid. The resultant pressure cone may promote brain herniation through the defect; this combined with normal growth of the brain and skull contributes to continued enlargement of the fracture [29]. In the majority of cases, fracture resolution relies on exposing the edges of the dura and creating a water-tight dural closure to normalize lines of intracranial pressure [29]. In the case presented here, we did not attempt to create a water-tight dural seal or repair the calvarial defect, rather relying on SIS as a dural substitute and polypropylene mesh and the temporalis musculature to provide a protective scaffold over the defect. We elected not to perform cranioplasty (e.g., with polymethylmethacrylate or titanium mesh) to minimize possible substrates for bacterial colonization. Use of polypropylene mesh also increases the risk of bacterial colonization; the use of a temporalis fascial graft instead would potentially have reduced this risk. However, the functional and cosmetic outcomes with the technique used in this patient were excellent; in the absence of direct focal trauma to that area we do not anticipate any drawbacks to this approach.

There was no evidence of residual abscess on the postoperative MRI. There was loss of brain parenchyma from the right cerebral hemisphere as well as hydrocephalus ex vacuo and T2 hyperintensity in the cortical gray matter suggestive of gliosis. The extra-axial heterogeneously contrastenhancing material is most consistent with a chronic subdural hematoma (CSDH), which refers to a fluid collection within the layers of the dura mater with preservation of the underlying arachnoid space [30]. Subdural hematomas tend 


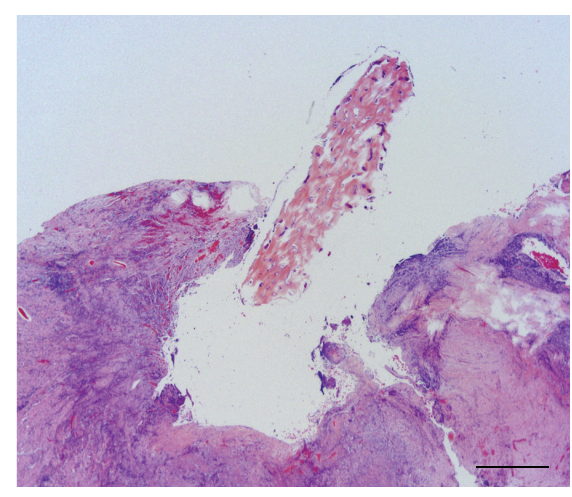

(a)

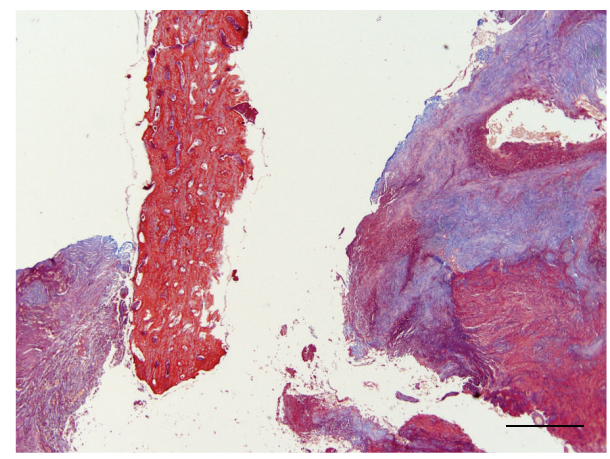

(c)

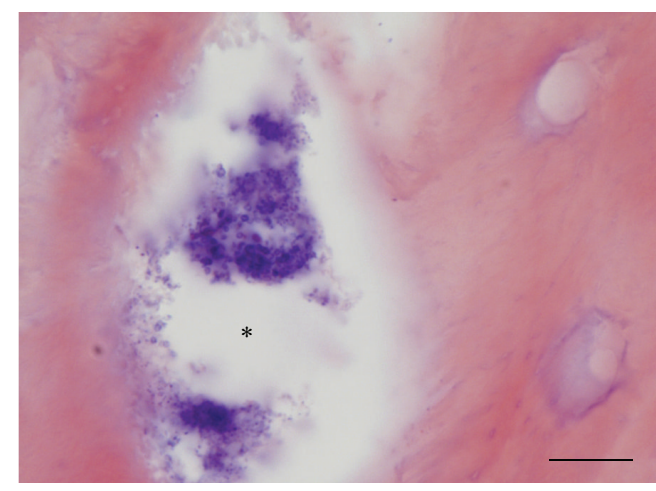

(b)

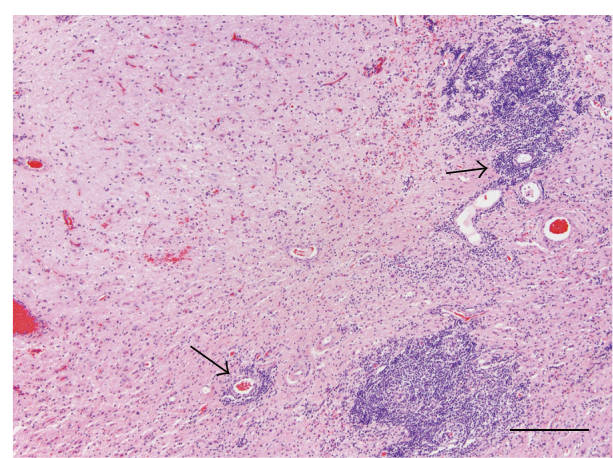

(d)

Figure 4: (a) Necrotic bone fragment surrounded by cavitated and inflamed neuropil. Hematoxylin and eosin $\times 2$, scale bar $=650 \mu \mathrm{m}$. (b) Gram-positive cocci within the marrow space (asterisk) of the necrotic bone fragment. Gram stain $\times 100$, scale bar $=8 \mu \mathrm{m}$. (c) Fibrosis (blue) of the neuropil surrounding the necrotic bone fragment (red). Masson's trichrome stain $\times 4$, scale bar $=400 \mu \mathrm{m}$. (d) Lymphoplasmacytic perivascular cuffs (arrows) and diffuse gliosis throughout the neuropil at the periphery of the sample. Hematoxylin and eosin $\times 10$, scale bar $=$ $175 \mu \mathrm{m}$.

to have a crescent shape and conform to dural attachments such as the falx cerebri (in contrast to epidural hematomas, which are biconvex in shape and can cross dural attachments but not calvarial sutures) [31]. The MR imaging characteristics of the different stages of SDH have been described [32]. CSDH are often hyperintense on T2W images and commonly have a layered appearance, as was appreciated in this case (Figure 5(d)) due to the development of internal septations and membranes joining the inner and outer membranes of the hematoma [33]. Rebleeding within the hematoma occurs frequently in people and also contributes to their heterogeneous appearance on MRI as well as to their potential for continued expansion [31]. It is common for both the membrane and the subdural collection to be enhanced following contrast administration, because the capsule surrounding the hematoma is a capillary-rich membrane that allows active exchange of contrast material $[31,34]$. It is therefore important to consider other causes of extra-axial contrast enhancement such as meningitis, subdural empyema, or en plaque meningioma in these cases.

$\mathrm{CSDH}$ is a potential complication of therapeutic procedures that significantly decrease the volume of the intracranial contents and thereby lead to intracranial hypotension; for example, CSF shunting is a well-recognized risk factor for CSDH in people $[30,35]$. This patient likely developed a postoperative subdural hematoma as a result of the rapid decrease in both tissue and CSF intracranial contents that occurred at the time of surgery. If not removed, subdural hematomas typically persist for months to over a year as they undergo compaction and fibrosis to eventually form a dense fibrous connective tissue layer much like the dura [31]. Surgical removal is recommended if the hematoma continues to increase in size on serial imaging or if the patient clinically deteriorates [30]. However, noncompressive mixtures of blood, CSF, and air are expected MRI findings in human patients after craniotomy; epidural collections are more common than subdural, but both are possible and typically do not require intervention [36]. Based on this, the lack of significant mass effect on the postoperative MRI, and the patient's continued clinical improvement, we did not perform further imaging to monitor the suspected SDH.

This case presents several interesting and novel findings: the potential for a brain abscess to have a chronic and insidious course prior to causing rapid neurologic deterioration, the importance of performing anaerobic bacterial culture and sensitivity in cases of suspected brain abscess, and the possibility of ventricular entrapment by an intracranial mass leading to partial hydrocephalus and intracranial 


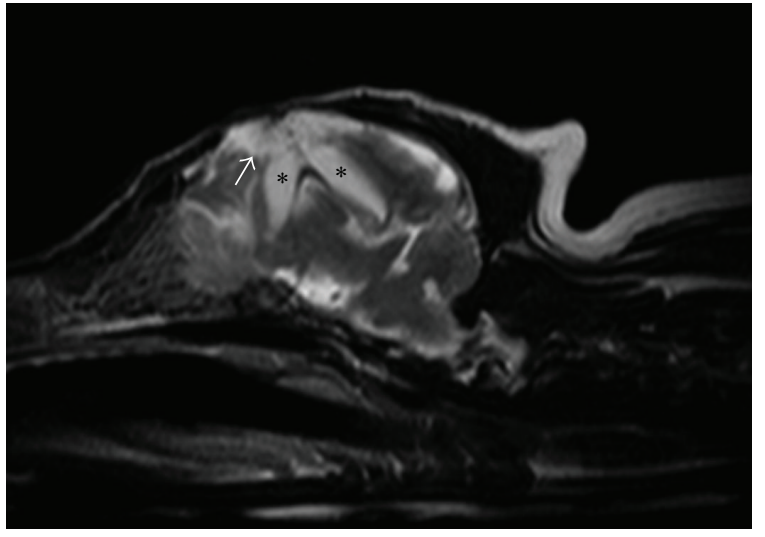

(a)

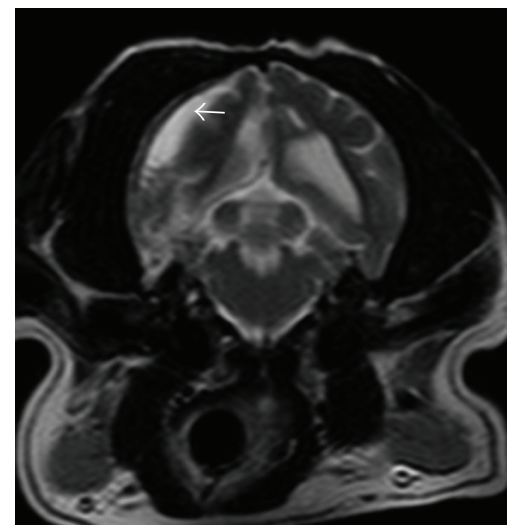

(c)

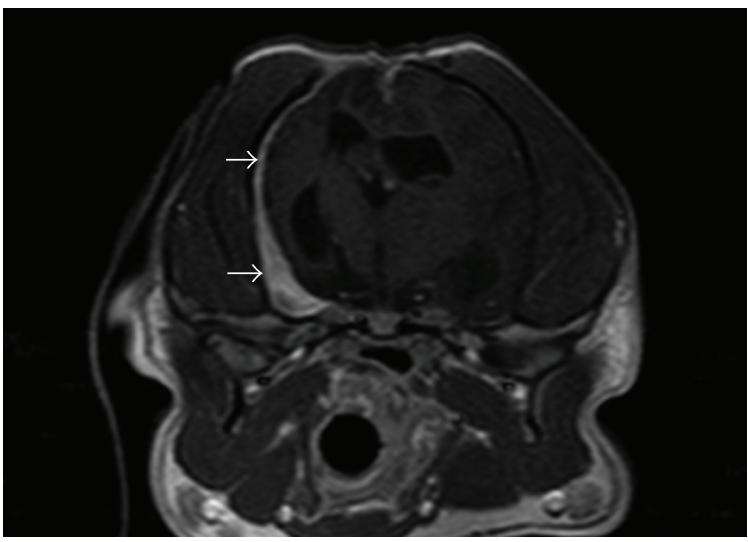

(f)

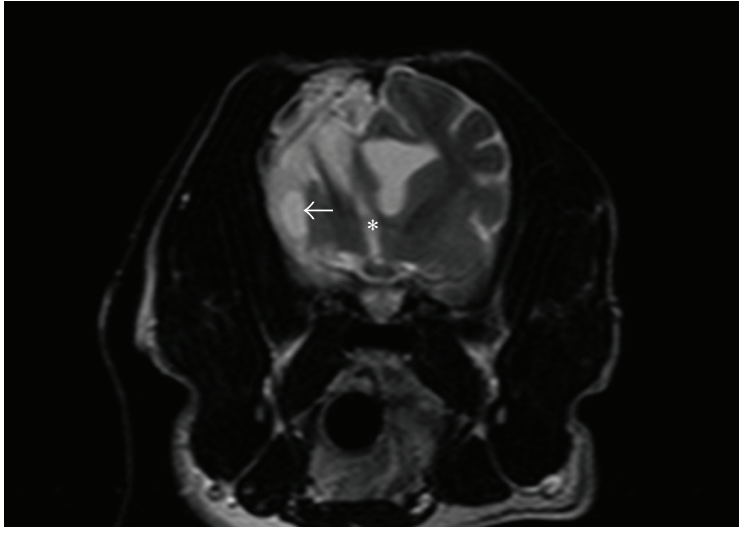

(b)

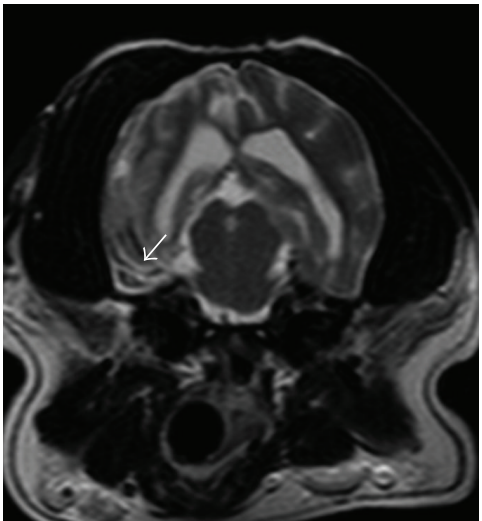

(d)

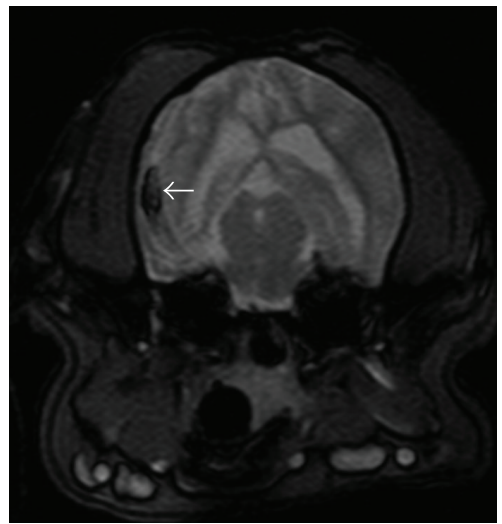

(e)

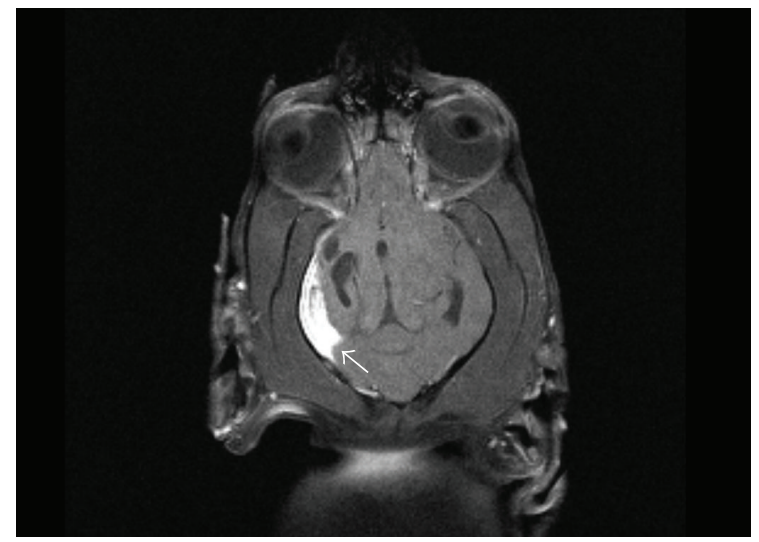

(g)

FIgure 5: Postoperative MR images of the head. (a) T2-weighted right parasagittal image. The shape of the right lateral ventricle is abnormal (asterisks), and there is T2-hyperintense tissue in the area previously occupied by the abscess (arrow). (b) T2-weighted transverse image at the level of the rostral third ventricle (asterisk). There is parenchymal loss on the right side, with resulting midline shift toward the right. There are extraventricular fluid pockets within the parenchyma suppressed on T2-weighted FLAIR imaging (arrow). (c) T2-weighted transverse image at the level of the caudal colliculi. There is a peripheral rim of predominantly T2-hyperintense material along the right side of the cerebrum (arrow). (d) T2-weighted transverse image at the level of the midbrain. The peripheral rim is seen ventrally and has mixed T2 signal and a layered appearance (arrow). (e) T2* -weighted transverse image at the level of the midbrain. Signal voids within the rim (arrow) are consistent with hemorrhage. (f) Postcontrast T1-weighted transverse image at the level of the thalamus. The peripheral rim strongly and, in most areas, uniformly contrast enhanced (arrows). (g) Postcontrast T1-weighted dorsal image at the level of the mesencephalic aqueduct. Note the sharply defined margin of the peripheral contrast-enhancing material along the tentorium cerebelli (arrow). 
hypertension. This case also documents for the first time a naturally occurring growing skull fracture in a dog. Finally, this case provides imaging findings consistent with a subdural hematoma that can be seen following removal of a large intracranial mass.

\section{Disclosure}

Preliminary results were presented at the 2nd Annual Southeastern Veterinary Neurology Group Conference, Athens, GA, October 2011.

\section{Conflict of Interests}

The authors declare that there is no conflict of interests regarding the publication of this paper.

\section{Acknowledgment}

The authors would like to thank Dr. Andy Shores for sharing his knowledge of growing skull fractures.

\section{References}

[1] A. L. Bilderback and D. Faissler, "Surgical management of a canine intracranial abscess due to a bite wound: Case Report," Journal of Veterinary Emergency and Critical Care, vol. 19, no. 5, pp. 507-512, 2009.

[2] E. G. H. Wouters, M. Beukers, and L. F. H. Theyse, "Surgical treatment of a cerebral brain abscess in a cat," Veterinary and Comparative Orthopaedics and Traumatology, vol. 24, no. 1, pp. 72-75, 2011.

[3] C. Costanzo, L. S. Garosi, E. N. Glass, C. Rusbridge, C. E. Stalin, and H. A. Volk, "Brain abscess in seven cats due to a bite wound: MRI findings, surgical management and outcome," Journal of Feline Medicine and Surgery, vol. 13, no. 9, pp. 672-680, 2011.

[4] I. Mateo, V. Lorenzo, A. Muñoz, and M. Pumarola, "Brainstem abscess due to plant foreign body in a dog," Journal of Veterinary Internal Medicine, vol. 21, no. 3, pp. 535-538, 2007.

[5] D. R. Enzmann, R. H. Britt, and R. Placone, "Staging of human brain abscess by computed tomography," Radiology, vol. 146, no. 3, pp. 703-708, 1983.

[6] R. H. Britt, D. R. Enzmann, and A. S. Yeager, "Neuropathological and computerized tomographic findings in experimental brain abscess," Journal of Neurosurgery, vol. 55, no. 4, pp. 590603, 1981.

[7] A. B. Haimes, R. D. Zimmerman, S. Morgello et al., "MR imaging of brain abscesses," American Journal of Roentgenology, vol. 152, no. 5, pp. 1073-1085, 1989.

[8] L. S. Klopp, J. T. Hathcock, and D. C. Sorjonen, "Magnetic resonance imaging features of brain stem abscessation in two cats," Veterinary Radiology and Ultrasound, vol. 41, no. 4, pp. 300-307, 2000.

[9] R. D. Zimmerman and A. B. Haimes, "The role of MR imaging in the diagnosis of infections of the central nervous system," Current Clinical Topics in Infectious Diseases, vol. 10, pp. 82-108, 1989.

[10] J. Pyhtinen, E. Pääkö, and P. Jartti, "Cerebral abscess with multiple rims on MRI," Neuroradiology, vol. 39, no. 12, pp. 857859, 1997.
[11] S.-Y. Yang and C.-S. Zhao, "Review of 140 patients with brain abscess," Surgical Neurology, vol. 39, no. 4, pp. 290-296, 1993.

[12] S. Stephanov, "Experience with multiloculated brain abscesses," Journal of Neurosurgery, vol. 49, no. 2, pp. 199-203, 1978.

[13] T.-M. Su, C.-M. Lan, Y.-D. Tsai et al., "Multiloculated pyogenic brain abscess: experience in 25 patients," Neurosurgery, vol. 52, no. 5, pp. 1075-1080, 2003.

[14] A. Danziger, H. Price, and M. M. Schechter, "An analysis of 113 intracranial infections," Neuroradiology, vol. 19, no. 1, pp. 31-34, 1980.

[15] N. Nathoo, S. S. Nadvi, P. K. Narotam, and J. R. van Dellen, "Brain abscess: management and outcome analysis of a computed tomography era experience with 973 patients," World Neurosurgery, vol. 75, no. 5-6, pp. 716-726, 2011.

[16] C.-H. Lu, W.-N. Chang, and C.-C. Lui, "Strategies for the management of bacterial brain abscess," Journal of Clinical Neuroscience, vol. 13, no. 10, pp. 979-985, 2006.

[17] S.-Y. Yang, "Brain abscess: a review of 400 cases," Journal of Neurosurgery, vol. 55, no. 5, pp. 794-799, 1981.

[18] H. Çavuşoglu, R. A. Kaya, O. N. Türkmenoglu, I. Çolak, and Y. Aydin, "Brain abscess: analysis of results in a series of 51 patients with a combined surgical and medical approach during an 11year period," Neurosurgical Focus, vol. 24, no. 6, p. E9, 2008.

[19] D. P. Calfee and B. Wispelwey, "Brain abscess," Seminars in Neurology, vol. 20, no. 3, pp. 353-360, 2000.

[20] S. Bhagwati, "A case of unilateral hydrocephalus secondary to occlusion of one Foramen of Monro," Journal of Neurosurgery, vol. 21, no. 3, pp. 226-229, 1964.

[21] H. L. Rekate, "A contemporary definition and classification of hydrocephalus," Seminars in Pediatric Neurology, vol. 16, no. 1, pp. 9-15, 2009.

[22] R. S. Maurice-Williams and M. Choksey, "Entrapment of the temporal horn: a form of focal obstructive hydrocephalus," Journal of Neurology Neurosurgery and Psychiatry, vol. 49, no. 3, pp. 238-242, 1986.

[23] P. Bret, S. Gharbi, F. Cohadon, and J. Remond, "Meningioma of the lateral ventricle: 3 recent cases," Neurochirurgie, vol. 35, no. 1, pp. 5-12, 1989.

[24] T. W. Eller and J. F. Pasternak, "Isolated ventricles following intraventricular hemorrhage," Journal of Neurosurgery, vol. 62, no. 3, pp. 357-362, 1985.

[25] A. P. Amar, S. Ghosh, and M. L. J. Apuzzo, "Ventricular tumors," in Youmans Neurological Surgery, R. H. Winn, Ed., vol. 1, pp. 1237-1263, Saunders, Philadelphia, Pa, USA, 5th edition, 2004.

[26] H. L. Rekate, "Hydrocephalus in children," in Youmans Neurological Surgery, R. H. Winn, Ed., vol. 3, pp. 3387-3404, Saunders, Philadelphia, Pa, USA, 5th edition, 2004.

[27] R. A. Lende and T. C. Erickson, "Growing skull fractures of childhood," Journal of Neurosurgery, vol. 18, no. 4, pp. 479-489, 1961.

[28] F. Goldstein, T. Sakoda, J. J. Kepes, K. Kavidson, and C. E. Brackett, "Enlarging skull fractures: an experimental study," Journal of Neurosurgery, vol. 27, no. 6, pp. 541-550, 1967.

[29] M. G. Muhonen, J. G. Piper, and A. H. Menezes, "Pathogenesis and treatment of growing skull fractures," Surgical Neurology, vol. 43, no. 4, pp. 367-373, 1995.

[30] J. K. Krauss, L. F. Marshall, and R. Weigel, "Medical and surgical management of chronic subdural hematomas," in Youmans Neurological Surgery, R. H. Winn, Ed., pp. 535-543, Saunders, St. Louis, Mo, USA, 6th edition, 2011. 
[31] V. L. Williams and J. P. Hogg, "Magnetic resonance imaging of chronic subdural hematoma," Neurosurgery Clinics of North America, vol. 11, no. 3, pp. 491-498, 2000.

[32] E. S. Fobben, R. I. Grossman, S. W. Atlas et al., "MR characteristics of subdural hematomas and hygromas at 1.5 T," American Journal of Roentgenology, vol. 153, no. 3, pp. 589-595, 1989.

[33] M. Kitagawa, M. Okada, H. Koie, K. Kanayama, and T. Sakai, "Magnetic resonance imaging and computed tomography appearance of chronic subdural haematoma in a dog," Australian Veterinary Journal, vol. 86, no. 3, pp. 100-101, 2008.

[34] S. Blitshteyn, L. L. Mechtler, and R. Bakshi, "Diffuse dural gadolinium MRI enhancement associated with bilateral chronic subdural hematomas," Clinical Imaging, vol. 28, no. 2, pp. 90-92, 2004.

[35] B. O’Neill, J. Wilberger, and A. Wilberger, "Pathophysiology of subdural hematomas," in Youmans Neurological Surgery, R. H. Winn, Ed., pp. 532-534, Saunders, St. Louis, Mo, USA, 6th edition, 2011.

[36] A. G. Sinclair and D. J. Scoffings, "Imaging of the post-operative cranium," Radiographics, vol. 30, no. 2, pp. 461-482, 2010. 

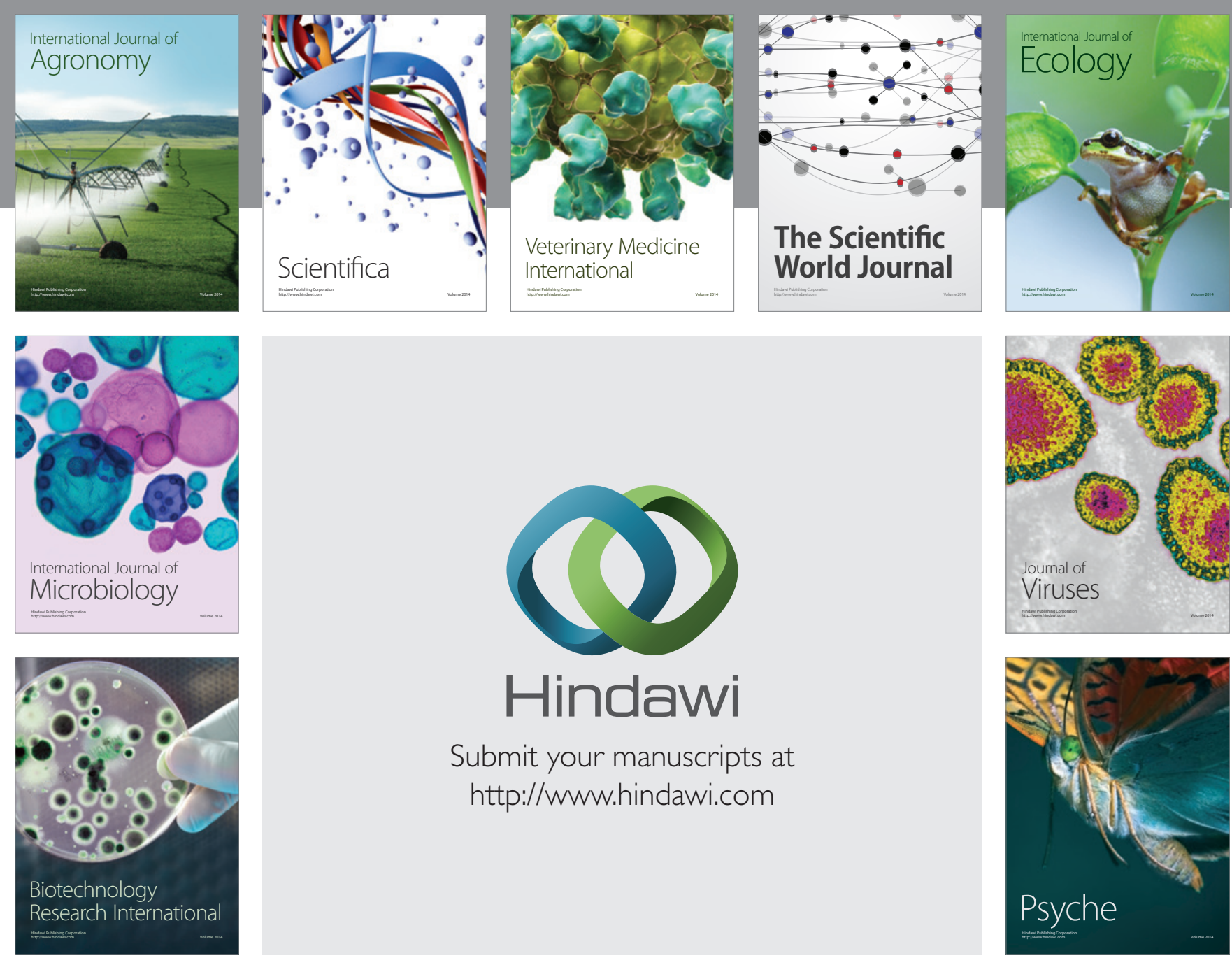

Submit your manuscripts at http://www.hindawi.com
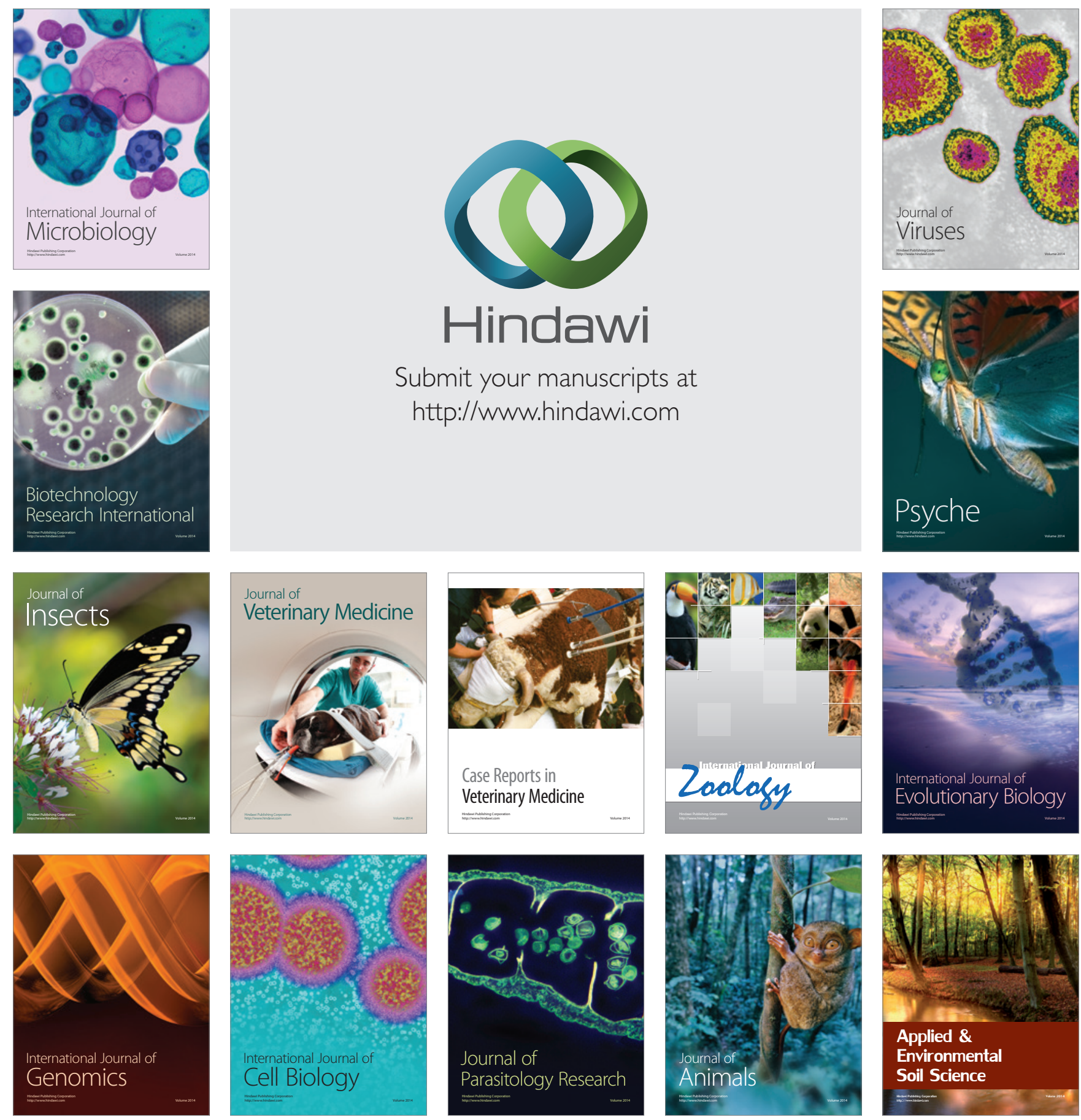